\title{
Myocardial infarction with normal coronary arteries: a case report and review of the literature
} Luigi Iuliano*1, Fausta Micheletta ${ }^{1}$, Alessandro Napoli² and Carlo Catalano ${ }^{2}$

\author{
Address: ${ }^{1}$ Department of Internal Medicine, Unit of Vascular Medicine, Sapienza University of Rome, Corso della Repubblica, 04100 Latina, Rome, \\ Italy and 2Department of Radiological Sciences, Sapienza University of Rome, via del Policlinico 155. 00161 Rome, Italy \\ Email: Luigi Iuliano* - luigi.iuliano@uniroma1.it; Fausta Micheletta - fausta.micheletta@uniroma1.it; \\ Alessandro Napoli - alessandro.napoli@uniroma1.it; Carlo Catalano - carlo.catalano@uniroma1.it \\ * Corresponding author
}

Published: 23 January 2009

Journal of Medical Case Reports 2009, 3:24 doi:10.1 186/1752-1947-3-24
Received: II October 2008

Accepted: 23 January 2009

This article is available from: http://www.jmedicalcasereports.com/content/3/1/24

(C) 2009 luliano et al; licensee BioMed Central Ltd.

This is an Open Access article distributed under the terms of the Creative Commons Attribution License (http://creativecommons.org/licenses/by/2.0), which permits unrestricted use, distribution, and reproduction in any medium, provided the original work is properly cited.

\begin{abstract}
Introduction: Although acute myocardial infarction is generally associated with obstructive coronary artery disease, myocardial infarction associated with normal coronary arteries is a wellknown condition. The overall prevalence rate of myocardial infarction with normal coronary arteries is considered to be low, varying from I\% to $2 \%$ depending on the definition of "normal" coronary arteries.
\end{abstract}

Case presentation: We describe here a case of a 49-year-old woman with a history of prior myocardial infarction who continued to be asymptomatic after a 10-year follow-up, in the absence of a high-risk profile for adverse outcomes. She was studied with multi-slice coronary computed tomography and whole-body angiography, which showed normal coronary and extra-coronary arteries.

Conclusion: This case report raises two important issues. First, the possible role of multi-slice computed tomography/coronary angiography in the risk- and prognosis assessment of patients with known or suspected coronary artery disease. Second, the important role played by long-term pharmacological therapy in patients with prior myocardial infarction and normal coronary arteries.

\section{Introduction}

Myocardial infarction (MI) with normal coronary arteries is a medical condition, which has been described in the literature for more than 30 years but is still a challenge in medical practice because of the lack of evidence-based medical data on its prognosis and on secondary prevention. The prevalence of MI with normal coronary arteries has always been considered low, varying between $1 \%$ and $12 \%$. However, it actually involves a considerable number of patients, calling for more clinical trials which specifically study this sub-population of patients affected by coronary heart disease (CHD) [1-3].
We report a case of a young female patient with a previous clinically diagnosed MI, who was asymptomatic in standard care therapy for a 10-year follow-up and has been shown to have normal coronary arteries by coronary multi-slice computed tomography (MSCT) and wholebody angiography.

\section{Case presentation}

A 49-year-old woman was referred to our department for a cardiological follow-up visit. She had a medical history of hypertension and previous MI, with no history of diabetes mellitus, hypercholesterolemia or premature coro- 
nary artery disease in her family. She did not smoke, take recreational drugs and was not on oestrogen replacement therapy.

Ten years earlier, she had an inferior MI treated with systemic thrombolysis, unfractioned heparin, aspirin, atenolol and intravenous nitroglycerine. During her hospitalization, the echocardiogram (ECG) revealed akinesis of the posterior-basal wall with an estimated ejection fraction of $50 \%$. Laboratory tests including serum glucose, lipids, blood count, liver-, kidney- and thyroidfunction tests were within normal limits. The day before her discharge, she had a stress ECG negative for inducible ischemia. The patient was discharged on the sixth day with a drug regimen of aspirin, atenolol, captopril, simvastatin and isosorbide dinitrate.

After the MI and prior to the visit to our department, the patient had been free from chest pain and dyspnea and had a normal exercise tolerance. She had periodical ECGs that showed the presence of akinesis of the posterior-basal wall with a mildly depressed systolic function. She reported a good control of arterial blood pressure with the ongoing therapy, including perindopril $5 \mathrm{mg} /$ day, hydroclhorotyazide $25 \mathrm{mg}$ /day and atenolol $25 \mathrm{mg} /$ day associated with aspirin and simvastatin $20 \mathrm{mg} /$ day.

A physical examination showed blood pressure of 135/80 $\mathrm{mmHg}$, heart rate of $64 \mathrm{bpm}$, BMI of $27 \mathrm{~kg} / \mathrm{m} 2$, waist circumference of $85 \mathrm{~cm}$ and no findings of congestive heart failure. The resting ECG showed a sinus rhythm at $62 \mathrm{bpm}$ and the presence of Q waves in II, III and aVF leads with no other abnormalities.

Laboratory tests revealed a total serum cholesterol of 160 $\mathrm{mg} / \mathrm{dl}$, HDL cholesterol of $55 \mathrm{mg} / \mathrm{dl}$, triglycerides of 78 $\mathrm{mg} / \mathrm{dl}, \mathrm{LDL}$ cholesterol of $89.6 \mathrm{mg} / \mathrm{dl}$ and fasting glycemia of $116 \mathrm{mg} / \mathrm{dl}$, with a normal OGTT and normal plasma homocysteine.

On the basis of her medical records concerning the MI, we confirmed the diagnosis of coronary artery disease (CAD) according to the recently published universal definition of MI [4].

For risk stratification, we studied the patient with noninvasive diagnostic tests, including echocardiography and a treadmill exercise test.

The ECG confirmed the presence of akinesis of the posterior-basal wall with a mildly depressed systolic function (LVEF 48\%) and the treadmill exercise test showed no signs of inducible ischemia.
Without any symptoms which suggest cardiac ischemia, the results from the non-invasive tests suggested no highrisk criteria for adverse outcomes for our patient. According to the guidelines by the American Heart Association/ American College of Cardiology (AHA/ACC), there was thus no indication for conventional coronary angiography.

Consequently, we decided to study her coronary arteries non-invasively. MSCT angiography was performed using a 64-slice computed tomography (CT) scanner (Sensation Cardiac 64; Siemens; Forchheim, Germany). The protocol included a post-coronary phase for whole-body angiography.

The MSCT angiographic analysis showed no coronary lesions (Figure 1), but the presence of a hypodense area involving more than $50 \%$ of the myocardial wall of the left ventricular inferior basal portion (Figure 2). No lesions were detected in the wholly explored extra-coronary arterial system (not shown).

The patient was sent to follow-up with a drug regimen of perindopril $5 \mathrm{mg} /$ day and hydrochlorothiazide $25 \mathrm{mg} /$ day. Aspirin, atenolol and simvastatin were discontinued. She was advised to lose weight and implement some lifestyle changes and was asked to repeat laboratory tests for a lipid profile before the next follow-up visit.

At the 6- and 12-month follow-up, the patient was free from chest pain, LDL-cholesterol was below $140 \mathrm{mg} / \mathrm{dl}$ without statin therapy and blood pressure was stable below 140/90 $\mathrm{mmHg}$.

\section{Discussion}

The overall prevalence rate of MI with normal coronary arteries is considered to be low, varying from $1 \%$ to $12 \%$, depending on the definition of "normal" coronary arteries, which usually includes no luminal irregularities (strict definition) or arteries with some degree of stenosis (in most cases, less than 30\% stenosis) [1-3]. The incidence seems to be strongly related to age and sex, with higher rates in young and female patients [5]. Thus, studies of women less then 45 years old, who have suffered acute MI, have showed normal coronary arteries angiographically in $7 \%$ to $32 \%$ of cases $[1,5,6]$. We have described a case of a young female patient with a history of prior MI who, after a 10-year follow-up, continued to be asymptomatic with no high-risk profile for adverse outcomes. She was studied by MSCT angiography, which showed normal coronary arteries.

This case report raises two important issues. First, the possible role of MSCT coronary angiography in risk- and prognosis assessment of patients with known or suspected 

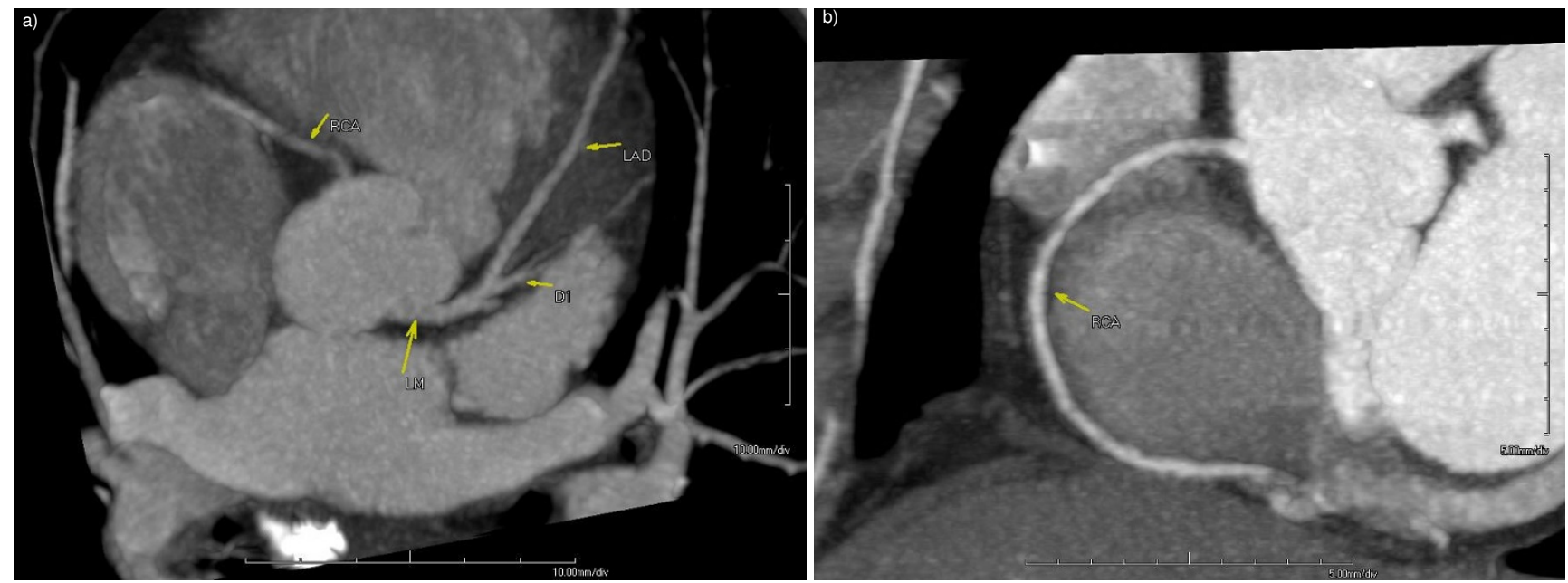

Figure I

Coronary computed tomography angiography shows absence of flow limiting stenosis in both left (I a) and right (Ib) coronary circulation. A tri-dimensional data set was reformatted using the maximum intensity projection technique. In Figure Ia, the image was taken along the anterior interventricular plane, allowing fine analysis of the left main as well as the left anterior descending coronary artery. A maximum intensity projection view also allows partial assessment of the origin of the right coronary artery and the first diagonal branch. Figure Ib: an image acquired along the right atrio-ventricular groove; the maximum intensity projection image clearly depicts the right circumflex artery up to the inferior portion.

CAD. Second, the role of long-term pharmacological therapy in patients with prior MI and normal coronary arteries.

MSCT provides high-resolution coronary angiograms non-invasively and has been demonstrated to be highly accurate in ruling out coronary atherosclerosis [7]. Its high

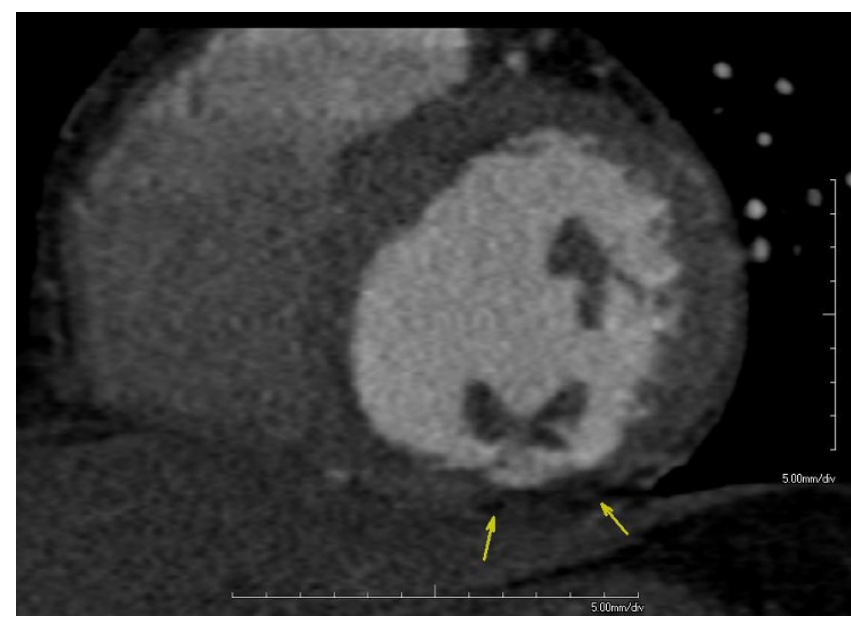

Figure 2

Multiplanar reformatted images generated along the conventional short cardiac axis, showing focal areas of myocardial wall thinning associated with hypodensity (arrows), suggestive for non-recent myocardial wall infarction. negative predictive value for exclusion of significant coronary artery stenosis, approaching $100 \%$, makes it an attractive method for the assessment of patients with known or suspected CAD and no high-risk profile for adverse outcomes; patients for whom conventional coronary angiography is not indicated according to current international guidelines. In particular, it might have an important application in patients for whom a CAD diagnosis was made on a clinical basis with no angiographic documentation.

We approached our patient according to a diagnostic algorithm based on the current AHA/ACC guidelines. Our patient had been asymptomatic since the MI event and had had no high-risk features for adverse outcomes in routine non-invasive tests. Medical history, clinical features and non-invasive tests did not provide specific diagnosis and prognosis, which allowed us only to continue the current medical therapy. According to the scheme provided by the current guidelines, we should have considered further imaging studies. However, in our patient, routine non-invasive imaging studies, such as nuclear stress testing or stress echocardiography would not have provided any new information for a better risk- and prognosis stratification. We considered the low age and the low cardiovascular risk profile of the patient at the time of acute MI, the absence of symptoms after the event and the absence of inducible ischaemia during the treadmill exercise test and decided that a non-invasive delineation of the pres- 
ence and severity of coronary atherosclerosis would allow insights for the further management of our patient.

MSCT angiography showed absolutely normal coronary arteries. The absence of atherosclerosis lesions in this patient was further confirmed in the extra-coronary arterial tree, thus providing a definitive diagnosis. The high negative predictive value of MSCT coronary angiography allowed us to definitely exclude CAD and the need for further diagnostic tests. Moreover, MSCT provided a more accurate prognosis for our patient for two main reasons. First, long-term prognosis in patients with MI and normal coronary arteries is much better compared to patients with coronary occlusive disease, especially in young and female patients $[2,5,8]$. Patients with normal coronary arteries have a good survival rate, around $90 \%$ at 3-7 years of follow-up in different studies, and a significant lower rate of reinfarction than patients with obstructive coronary disease $[2,5,8]$. Second, it has been recently demonstrated that MSCT provides independent prognostic information on baseline clinical risk factors in patients with known or suspected CAD, showing an excellent prognosis in patients with normal coronary arteries [9].

The diagnosis of prior MI with normal coronary arteries raises a second important issue concerning the application of AHA/ACC secondary prevention guidelines for CHD. According to these guidelines, patients who have had MI should receive indefinitely a drug regimen including an antiplatelet agent, a beta-blocker, a statin with an LDL-C goal of $<100 \mathrm{mg} / \mathrm{dl}$ or $<70 \mathrm{mg} / \mathrm{dl}$, and an aceinhibitor with a blood pressure goal $<140 / 90 \mathrm{mmHg}$ [10]. As stated in the paper, cases covered by these guidelines include patients with established coronary and other atherosclerotic vascular disease. Thus, cases with prior MI and normal coronary arteries are actually not covered by these guidelines, and there are no other indications on the management of these patients in the literature.

The estimated annual incidence of MI, new and recurrent in the US is 865000 , and among these, about 350000 are women (11). Among these women, the above-mentioned $7 \%$ to $32 \%$ with normal coronary angiography translate into 24500 to 112000 women with acute MI and normal coronary arteries annually in the US alone. This sub-population of patients affected by CHD is significant and the lack of randomized clinical trials, comparing therapies for the reduction of adverse cardiac events in patients with MI and normal coronary arteries, makes their management challenging. It is difficult to find a physio-pathological rationale that allows us to transfer secondary prevention guidelines from patients with established coronary artery disease to patients with normal coronary arteries.
On the other hand, these patients are young people with good prognoses, which implies that a long-term multidrug therapy should be carefully considered in terms of cost-benefit analysis. Despite an undemonstrated improvement of the prognosis, a multi-drug regimen including antiplatelet agents, statins, beta-blockers and ace-inhibitors would expose our patient to well-known adverse effects and imply a considerable economic cost.

According to the clinical features and the diagnostic test results from our patient, we decided to retain the aceinhibitor because of arterial hypertension and to discontinue aspirin, statin and beta-blocker because of the absence of coronary atherosclerotic disease, hypercholesterolemia and symptoms reflecting inducible ischaemia.

\section{Conclusion}

MSCT coronary angiography can have an important role in the risk and prognosis assessment of patients with known or suspected CHD. Large-scale randomized trials need to be conducted to determine an optimal secondary prevention strategy for patients with MI and normal coronary arteries, who constitute a large sub-population of CHD patients.

\section{Abbreviations}

AHA/ACC: American Heart Association/American College of Cardiology; CAD: coronary artery disease; CHD: coronary heart disease; CT: computed tomography; ECG: echocardiogram; MI: myocardial infarction; MSCT: multislice computed tomography; OGTT: oral glucose tolerance test.

\section{Consent}

Written informed consent was obtained from the patient for publication of this case report and accompanying images. A copy of the written consent is available for review by the Editor-in-Chief of this journal.

\section{Competing interests}

The authors declare that they have no competing interests.

\section{Authors' contributions}

LI was in charge of the patient clinically and was a major contributor in writing the manuscript. MF collected clinical data and reviewed the literature. AN prepared a CT angiography protocol to study in a single-setting coronary and whole-body arterial system. CC analyzed and interpreted the imaging studies. All authors read and approved the final manuscript.

\section{Acknowledgements}

The authors thank Tracie Dornbusch for editing the manuscript. 


\section{References}

I. Alpert JS: Myocardial infarction with angiographically normal coronary arteries. Arch Intern Med 1996, 154(3):265-269.

2. Da Costa A, Isaaz K, Faure E, Mourot S, Cerisier A, Lamaud M: Clinical characteristics, aetiological factors and long-term prognosis of myocardial infarction with an absolutely normal coronary angiogram; a 3-year follow-up study of 91 patients. Eur Heart J 200I, 22(16): |459- 1465.

3. Bugiardini R, Bairey Merz CN: Angina with "normal" coronary arteries: a changing philosophy. Jama 2005, 293(4):477-484.

4. Thygesen K, Alpert JS, White HD: Universal definition of myocardial infarction. Eur Heart J 2007, 28(20):2525-2538.

5. Zimmerman FH, Cameron A, Fisher LD, Ng G: Myocardial infarction in young adults: angiographic characterization, risk factors and prognosis (Coronary Artery Surgery Study Registry). J Am Coll Cardiol 1995, 26(3):654-66I.

6. Legrand V, Deliege M, Henrard L, Boland J, Kulbertus H: Patients with myocardial infarction and normal coronary arteriogram. Chest 1982, 82(6):678-685.

7. Leschka S, Alkadhi H, Class A, Desbiolles L, Grunenfelder J, Marincek $B$, Wildermuth S: Accuracy of MSCT coronary angiography with 64-slice technology: first experience. Eur Heart J 2005, 26(I5): I 482-I 487.

8. Raymond R, Lynch J, Underwood D, Leatherman J, Razavi M: Myocardial infarction and normal coronary arteriography: a 10 year clinical and risk analysis of $\mathbf{7 4}$ patients. J Am Coll Cardiol | 988, I I(3):47| - 477.

9. Pundziute G, Schuijf JD, Jukema JW, Jukema JW, Boersma E, de Roos $A$, Wall EE van der, Bax J]: Prognostic value of multislice computed tomography coronary angiography in patients with known or suspected coronary artery disease. J Am Coll Cardiol 2007, 49(I):62-70.

10. Smith SC Jr, Allen J, Blair SN, et al.: AHA/ACC guidelines for secondary prevention for patients with coronary and other atherosclerotic vascular disease: 2006 update: endorsed by the National Heart, Lung, and Blood Institute. Circulation 2006, I I 3( (19):2363-2372.

II. Heart Disease and Stroke - 2007 update, American Heart Association [http://www.americanheart.org/statistics]

Publish with Biomed Central and every scientist can read your work free of charge

"BioMed Central will be the most significant development for disseminating the results of biomedical research in our lifetime. "

Sir Paul Nurse, Cancer Research UK

Your research papers will be:

- available free of charge to the entire biomedical community

- peer reviewed and published immediately upon acceptance

- cited in PubMed and archived on PubMed Central

- yours - you keep the copyright

Submit your manuscript here:

http://www.biomedcentral.com/info/publishing_adv.asp
BioMedcentral 